\title{
Music Therapy in US Corrections :
}

\section{Philosophy, Practice, and Protocols of Music Therapists Working with Incarcerated People in the US}

\author{
Lorna E. Segall ${ }^{1 *}$, Olivia S. Yinger ${ }^{2}$ \\ 1 University of Louisville, USA \\ 2 University of Kentucky, USA \\ *lornasegall@yahoo.com
}

Received: 19 August 2019 Accepted: 24 August 2021 Published: 1 March 2022

Editor: Helen Brenda Oosthuizen Reviewers: Sarah Pitts, Lars Tuastad

\begin{abstract}
Although the United States has the highest incarceration rate in the world and music therapy has existed in U.S. correctional facilities for almost a century, little is known about music therapists who provide services to people who are incarcerated. Exploration in this area is important as it could help inform clinical practice, music therapy curriculum, and potentially influence policy in the treatment of inmates. This study explores the philosophy, practices, and protocols of music therapists working in the U.S. corrections system. Board-certified music therapists $(N=542)$ completed an online survey in which they indicated their reasons for working or not working in corrections. Participants who worked in corrections $(n=52)$ answered additional questions about their religious beliefs and political affiliation, music therapy practices in corrections, and protocols at their facility related to provision of music therapy services. The results of this survey revealed differences in demographic variables for music therapists who worked in prisons, who were significantly more likely to be men, $\chi^{2}(1)=6.57, p=.015$, or under-represented racial minorities, $\chi^{2}(1)=5.82, p=$ .021 , than music therapists who did not work in prisons, who were representative of music therapists in the U.S. (almost $90 \%$ white women). The majority of respondents who worked in corrections reported they were Democrats and more than half reported that their religious and/or spiritual views did not influence their decision to work in corrections. Music therapists most frequently addressed emotional awareness, impulse control, and self-awareness through music listening, discussion, and playing instruments. We discuss results in the context of current efforts to improve rehabilitation and treatment services within the U.S. correctional system.
\end{abstract}

Keywords: music therapy, corrections, prison

\section{Introduction}

The practices of imprisonment for controlling crime worldwide are as diverse as the people who inhabit correctional facilities. Without question, however, the United 
States (U.S.) remains an outlier for its high rates of incarceration and total number of incarcerated people (Jacobsen et al., 2017). In fact, 1 out of 5 incarcerated people in the world is incarcerated in the U.S. (Sawyer \& Wagner, 2020). Nearly seven million people are under supervision of the U.S. correctional system. These people are either currently incarcerated or are serving their sentences via parole or probation. Of those, almost 2.3 million people (or 698 per 100,000 residents) are held in facilities such as state and federal prisons, juvenile correctional facilities, jails, and immigration detention facilities. In the U.S., poverty and race play key roles in mass incarceration; people who are incarcerated are disproportionately poor, and people of color. In particular, Black Americans are overrepresented among incarcerated people (Sawyer \& Wagner, 2020). Considering the U.S. high incarceration rate and a century long history of music therapy within its system, it would seem valuable to know more about the music therapists working within prison walls to potentially increase service offering, advocate for treatment, and inform the music therapy profession on the current state of music therapy with this population. The focus of this study was to better understand the roles that music therapists play in the U.S. correctional system when working with incarcerated people.

\section{Review of Literature}

There is much debate in the U.S. about the purpose of the correctional system that is based in part on ideological differences related to opinions of crime causation (Sims, 2003). The traditionally conservative view (often associated with the Republican party in the U.S.) is that individuals who commit crimes are acting on the basis of free will and lack self-control or moral values, whereas the traditionally liberal view (frequently held by members of the Democratic party in the U.S.) is that crime results from external forces like inequality and discrimination (Sims, 2003). Although not always the case, proponents of the conservative view tend to believe that the purpose of the correctional system is to punish and keep society safe from dangerous people, whereas proponents of the liberal view tend to advocate for rehabilitating offenders and reforming a system that is unjust (Sims, 2003). In spite of these relationships between political affiliation, political ideology, and correctional ideology, there is evidence that support for rehabilitation and alternatives to incarceration has increased in recent years even in deeply conservative, Republican regions of the U.S. (Thielo et al., 2015).

Religious beliefs also influence views on criminal punishment, sometimes to a greater degree than political affiliations (Thomson \& Froese, 2017). People who believe in a punitive God tend to favor harsher punishments for criminals, whereas people whose religious beliefs include a greater focus on forgiveness tend to support rehabilitation (Evans \& Adams, 2003). Of course these trends do not hold for all individuals.

Because people who are incarcerated in the U.S. have lost certain freedoms and people working in corrections (most of whom are white and male, according to Burkhardt, 2017) are in an elevated position of power, the correctional ideologies of correctional employees are of interest to researchers. In a meta-analysis on predictors of correctional officers' attitudes and behaviors, Maahs and Pratt (2001) found that non-white correctional officers and those who had less role conflict more often held attitudes supportive of rehabilitation. Although race appeared to be related to correctional ideology for correctional officers, researchers found, in another study, that the same relationship did not hold true for prison chaplains. Sundt and Cullen (2002) explored the correctional orientation of prison chaplains and found that correctional approaches were influenced by their age, religious philosophy, and dynamics of the work environment, but not by race. The investigators found that chaplains who felt like their work was a calling from God and those who believed in religious forgiveness were less likely to support punishment for inmates. Sundt and Cullen also found that the majority of chaplains thought that treatment and rehabilitation for offenders were at least as im- 
portant as punishment. Thus, race, religious beliefs, age, political affiliations, and work dynamics may relate to correctional ideology to differing degrees depending on the profession of correctional employees.

In spite of the differences in correctional ideologies related to political affiliations, there have been recent bipartisan efforts at prison reform in the U.S. (Dolan, 2020). One of the aims of the Formerly Incarcerated Reenter Society Transformed Safely Transitioning Every Person (FIRST STEP) Act, which the U.S. Senate and House of Representatives voted overwhelmingly to pass in 2018, was to prepare people who are incarcerated for release from prison and reduce the likelihood that they will return to prison (Formerly Incarcerated Reenter Society Transformed Safely Transitioning Every Person Act, 2018). Dramatic changes in corrections reform are also taking place at the state level to reduce incarceration, support the re-entry process, and to minimize the long-term consequences of incarceration in the U.S. (Porter, 2020).

Accessing adequate mental health care is one important step toward rehabilitation for people who are incarcerated. According to the authors of a study on the prevalence of mental illness in a state prison system, "correctional facilities have become a front line for mental health care" (Al-Rouson et al., 2017, p. 342). Another important step toward rehabilitation is acknowledging the incarcerated female population. While much of the corrections literature explores the incarceration of disadvantaged Black men, women are the fastest growing population in the corrections system (Beall, 2018). The U.S. alone houses one-third of the world's incarcerated female population, although women only represent $5 \%$ of the global incarcerated population (Beall, 2018). In addition to needs related to mental health and gender, the aging population continues to grow and offers a unique set of needs to care for individuals aging in prison (Carson \& Sabol, 2016).

Music therapy's evidence-based research supports its efficacy in a variety of settings and populations such as rehabilitation (American Music Therapy Association, 2006), mental illness, the aging population, and the growing rate of women who are incarcerated. Thus, support for music therapy programs in the U.S. prison system continues to grow. It would seem valuable to learn more about the philosophies and practices of the music therapists who choose to work in correctional facilities. This knowledge could inform the practice of current music therapists, influence curriculum design for music therapists in training, and provide meaningful support for policy development in the service provisions for inmates.

\section{Music Therapy in Correctional Facilities}

Research on the use of music therapy with people who are incarcerated has been conducted in several countries around the world, including Australia (Daveson \& Edwards, 2001), China (Chen et al., 2015), the Netherlands (Hakvoort et al., 2015), Norway (Gold et al., 2014), the U.K. (Odell-Miller et al., 2019), and the U.S. (Cohen, 1987). In the U.S., as early as 1923, Willem van de Wall's publication, Music in Correctional Institutions suggests music is a worthwhile method of rehabilitation because of its capacity to create an environment of expressive and thoughtful development and to provide opportunities for group work, self-expression, and self-discipline (van de Wall, 1923). Several scholars have published case reports on music therapy practices with incarcerated men (Nolan, 1983), violent offenders (Cohen, 1987), and psychiatric patients residing in prisons (Thaut, 1989). More recently, researchers have explored the effects of music therapy on mental health issues of people who are incarcerated related to anxiety, depression, self-esteem (Chen et al., 2015; Chen et al., 2016; Gold et al., 2014) anger management, and coping techniques (Hakvoort et al., 2015). Tuastad and O'Grady (2013) examined how music therapy enables those who are incarcerated to maintain their humanity while existing within the dehumanizing prison environment. Group singing and public performance as a therapeutic intervention has also been explored as it relates to enhancing levels of executive function (Segall, 2016) and increasing inmates' potential to benefit from therapeutic interventions (O'Grady et al., 2015). 
Music therapy as a method of assisting former inmates with the re-entry process has also been explored (Tuastad \& Stige, 2015) as well as the impact of music making as an everyday occurrence in prison culture (Hjørnevik \& Waage, 2019), and the longterm effects of music therapy treatment (Gold et al., 2020). Although an encouraging body of evidence exists exploring the outcomes of music therapy with people who are incarcerated, little is known about the music therapists who are providing these services, and there has been little research conducted in the past 20 years on the use of music therapy in the U.S. correctional system. As the field of music therapy is constantly evolving to meet the needs of its growing body of patients, it's important to examine every way in which music therapists are providing services. This is important as it informs curricula for music therapists in training, informs clinical practice for those currently working in the field, and can also support policy and decision making for individuals who are currently incarcerated and the profession of music therapy.

The vast majority of music therapists practicing in the U.S. are white women (AMTA, 2018), and this trend has not changed greatly in the past few decades (Silverman \& Furman, 2014). In a study of membership trends in AMTA from 1998 to 2009, Silverman and Furman remarked that, with regard to gender, "data were extraordinarily consistent" (2014, p. 107). Over the course of that decade, about $87 \%$ of the membership was female and $13 \%$ was male. Assuming that the percentage of music therapists who are female has remained consistent over the past 20 years, the percentage of participants in Codding's (2002) study who were male (29\%) seems to be higher than one would expect based on the AMTA workforce analysis. Since most correctional employees are men (Burkhardt, 2017) and researchers have found relationships between race and correctional ideology (Maahs \& Praat, 2001), it would be of interest to note whether music therapists working in correctional institutions are representative of the larger population of music therapists with regard to race/ethnicity and gender, and whether they are representative of the inmates they serve. In light of evidence that unintentional bias from white counselors and psychotherapists may interfere with development of therapeutic alliance with clients of color (Vasquez, 2007), it would be of particular interest to know the race/ethnicity of music therapists practicing in correctional settings, since people of color are overrepresented among incarcerated people in the U.S. (Sawyer \& Wagner, 2020). Furthermore, since correctional ideologies are often related to religious beliefs and political affiliations, it would be of interest to know how music therapists describe their political affiliation and the degree to which their religious beliefs influenced their decision to work in corrections. This could help inform clinical practice by identifying the degree to which music therapists are at risk for issues that face other correctional workers like role conflict, which researchers studying prison chaplains and correctional workers have linked with stress, personal issues, and burnout (Allard et al., 2003; Denney, 2017; Sundt \& Cullen, 2002).

Identifying reasons why music therapists in the U.S. choose to work in corrections-and reasons why they choose not to work in corrections-could help provide insight into factors related to correctional ideology (i.e., if music therapists choose to work in corrections to assist with rehabilitation and reform). It could also help provide information for music therapy educators and policy makers regarding the degree to which music therapists in the U.S. have opportunities to work in corrections and potentially help explain the reason there has been so little written about music therapy in the U.S. correctional system in the past 20 years given the high rates of incarceration in the U.S.

The most recently published information about music therapy practices in corrections is from a survey published by Codding in 2002 that reported the demographics, conditions of employment, service provision, assessment, therapeutic objectives, and related values of music therapists. Given changes that have taken place in correctional institutions in the U.S. and within the music therapy profession over the past 20 years there is a need for updated information about music therapy practices in the U.S. correctional system. 
The purpose of this study was to explore current music therapy practices and protocols in U.S. corrections, as well as the philosophies of music therapists working in these correctional settings, using a survey approach with both descriptive and comparative design features. More specifically, we aimed to gain a broad overview of this area of practice in an effort to motivate further research in this area. In addition to updating information provided in Codding's study, the authors of the present study sought to investigate why music therapists chose to work, or not work, in corrections; how safe music therapists feel working in correctional settings; and whether demographic representation of those working in prisons differs from music therapists who work with other populations.

\section{Research Questions}

1. Demographic information: Are music therapists who work in correctional facilities representative of the overall population of music therapists with regard to race/ethnicity and gender?

2. Philosophy: Why do music therapists choose to work in corrections? Why not? What trends exist among music therapists who work in corrections related to political affiliation and influence of religious beliefs on their decision to work in corrections?

3. Practice: What types of musical experiences, goals, and treatment models do music therapists use when working in corrections?

4. Protocols: What are music therapy protocols in correctional facilities where music therapists work as related to issues of security, referrals, termination of treatment, group size, and frequency of sessions?

\section{Method}

The Institutional Review Board at the University of Kentucky reviewed this study and confirmed that it met criteria for exemption, since it was a survey in which no identifying information about participants was collected. This survey was administered via the secure REDCap server. Data on the REDCap is securely kept on biomedical informatics servers, the secure data center run by the Institute for Pharmaceutical Outcomes Policy (IPOP).

Participants were board-certified music therapists living in the U.S. who identified themselves as willing to receive emails from the Certification Board for Music Therapists (CBMT). In September 2017, the researchers e-mailed surveys to 6,300 board-certified music therapists using REDCap. The survey remained open for 10 weeks (from early-September to mid-November 2017) and researchers emailed survey reminders two and four weeks after the initial email.

Inspired by Codding's (2002) survey, the survey used in this study included 27 questions about: a) demographic information, b) therapist's values related to work in corrections, c) music therapy practices in correctional facilities, d) information about facilities, clients, and security, and e) information about provision of music therapy services in corrections. Participants were asked to answer questions via Likert-type rating scales, closed-ended questions, and narrative. Answers formatted with a Likert-type response consisted of three-point scales indicating responses such as: (1) not at all, (2) somewhat, or (3) greatly; and (1) did not have the opportunity, (2) did not want to, or (3) didn't think it was important.

We used Pearson's chi-square tests (Field, 2013) to explore potential differences between music therapists who worked in prisons and music therapists who did not work in prisons with regard to gender and race/ethnicity. For other demographic information and data related to philosophy, practice, and protocols, we reported descriptive data (frequencies and percentages). 


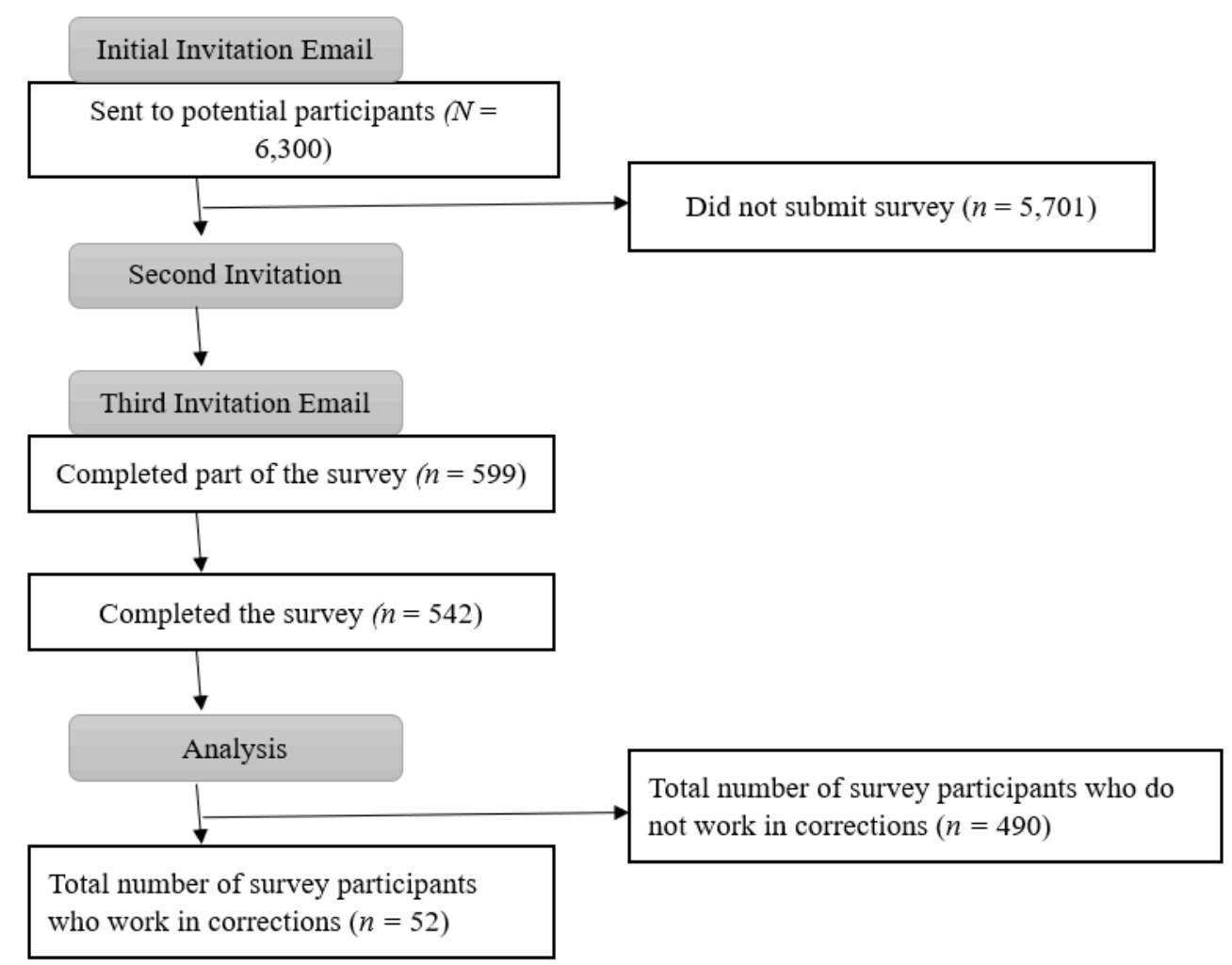

Figure 1

Participant Flowchart

\section{Results}

Of the 6,300 music therapists invited to participate, 599 responded and 542 returned completed surveys, a response rate of $9 \%$. See Figure 1 for a participant flow chart. Participants were mostly female $(n=470,87 \%)$, white $(n=393,88 \%)$, and 20-39 years old $(n=339,65 \%)$. Respondents represented all of AMTA's regions, with the most participants reporting that they practice music therapy in the Great Lakes $(n=$ $122,24 \%)$, Southeastern $(n=107,21 \%)$, and Mid-Atlantic $(n=98,20 \%)$ regions of the U.S. Participants tended to be relatively new to the profession, with $64 \%$ ( $n=$ 336) having practiced for 10 years or fewer.

\section{Research Question 1: Demographic Variables}

Table 1 shows the gender and race/ethnic origin of participants who worked in prison compared to those who did not. Two Pearson's chi-square tests revealed significant differences between music therapists who worked in prisons and music therapists who did not work in prisons with regard to gender, $\chi^{2}(1)=6.57, p=.015$, and ethnic ori$\operatorname{gin} \chi^{2}(1)=5.82, p=.021$. Among music therapists who reported they did not work in prisons, females outnumbered males by an odds ratio of 7.98, whereas female music therapists working in prisons only outnumbered male music therapists working in prisons by an odds ratio of 3.25. Among music therapists who did not work in prisons, white music therapists outnumbered under-represented minorities by an odds ratio of 7.71, whereas white music therapists working in prisons only outnumbered under-represented minorities by an odds ratio of 3.18. Based on the odds ratios, the odds of a music therapist being male or non-white were 2.46 and 2.42 times greater, respectively, if one practiced music therapy in prison settings. Figure 2 and Figure 3 show key differences in gender and ethnic origin for music therapists who worked in prisons and music therapists who did not. 
Table 1

Participants' Demographic Information: Gender and Race/Ethnic Origin

\begin{tabular}{|c|c|c|c|c|c|c|}
\hline & \multicolumn{6}{|c|}{ Work in prisons? } \\
\hline & \multicolumn{2}{|c|}{ Total } & \multicolumn{2}{|c|}{ Yes } & \multicolumn{2}{|l|}{ No } \\
\hline & $n$ & $\%$ & $n$ & $\%$ & $n$ & $\%$ \\
\hline \multicolumn{7}{|l|}{ Gender } \\
\hline Female & 470 & 87.2 & 39 & 76.5 & 431 & 88.3 \\
\hline Male & 66 & 12.2 & 12 & 23.5 & 54 & 11.1 \\
\hline Trans & 2 & 0.4 & 0 & 0.0 & 2 & 0.4 \\
\hline Other & 1 & 0.2 & 0 & 0.0 & 1 & 0.2 \\
\hline Total & 539 & 100.0 & 51 & 100.0 & 488 & 100.0 \\
\hline \multicolumn{7}{|l|}{ Race/ethnic origin } \\
\hline African American & 12 & 2.4 & 3 & 6.3 & 9 & 2.0 \\
\hline American Indian or Alaskan Native & 1 & 0.2 & 1 & 2.1 & 0 & 0.0 \\
\hline Asian/Asian American & 20 & 4.0 & 4 & 8.3 & 16 & 3.6 \\
\hline Caucasian/White & 393 & 87.9 & 35 & 72.9 & 428 & 86.5 \\
\hline Hispanic/Latinx & 15 & 3.0 & 1 & 2.1 & 14 & 3.1 \\
\hline Multiracial & 13 & 2.6 & 2 & 4.2 & 11 & 2.5 \\
\hline Pacific Islander & 1 & 0.2 & 0 & 0.0 & 1 & 0.2 \\
\hline Other & 5 & 1.0 & 2 & 4.2 & 3 & 0.7 \\
\hline Total & 495 & 100.0 & 48 & 100.0 & 447 & 100.0 \\
\hline
\end{tabular}

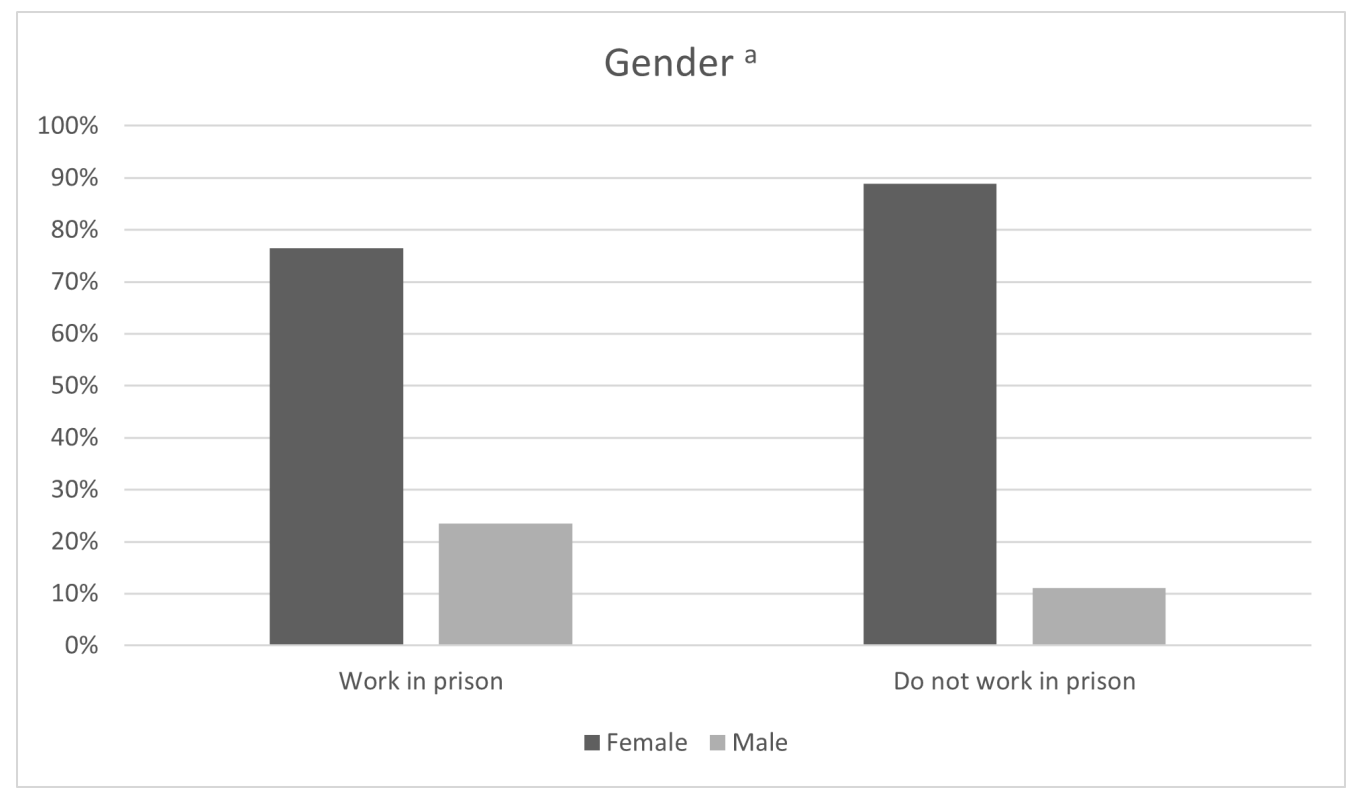

\section{Figure 2}

Differences in Gender of Music Therapists Who Work in Prisons and Music Therapists Who Do Not

a Because there were fewer than 5 participants who identified as transgender or other, it was not possible to include them in Pearson's chi-square analyses. 


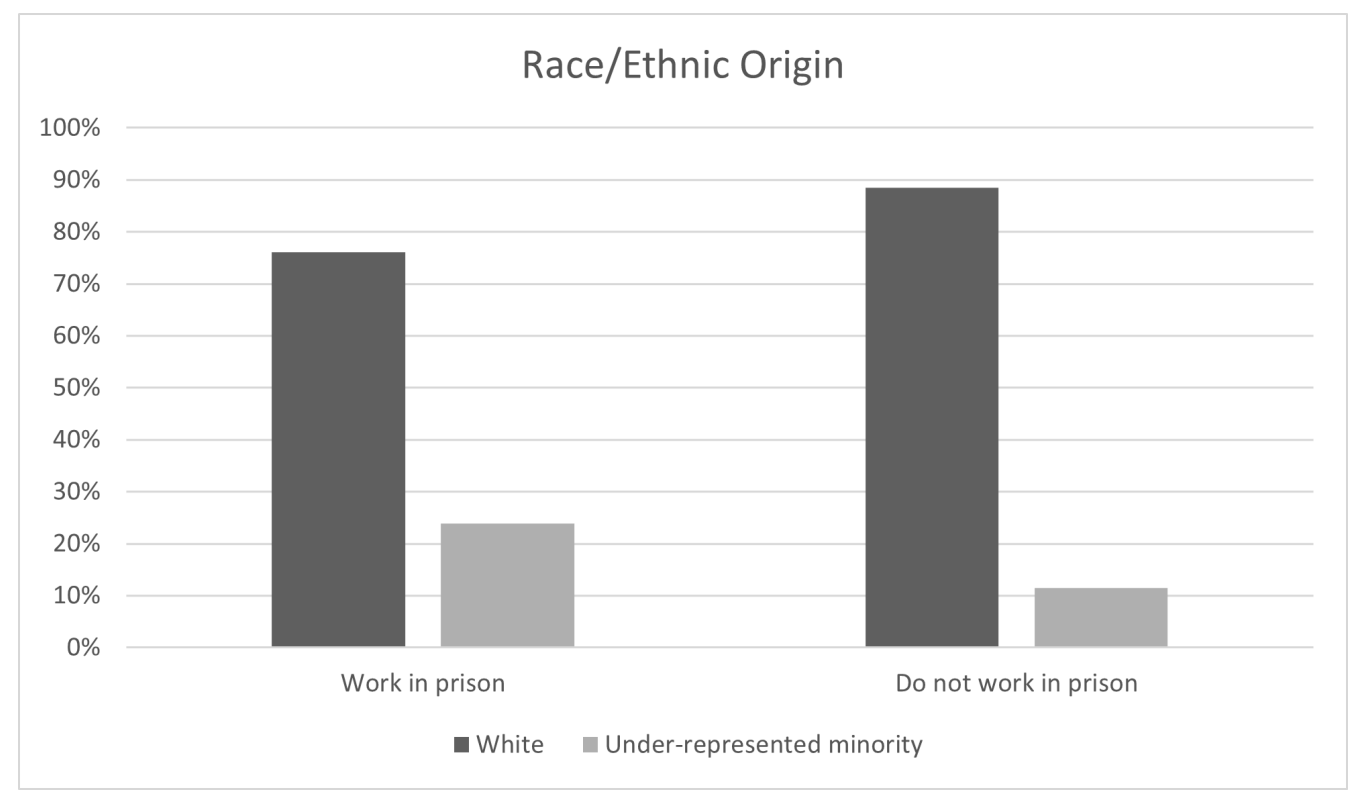

Figure 3

Differences in Race/Ethnic Origin of Music Therapists Who Work in Prisons and Music Therapists Who Do Not

\section{Research Question 2: Philosophy}

Music therapists who reported not having worked in corrections $(n=490)$ frequently reported that they did not have the opportunity to do so $(n=340,69 \%)$. Others reported that they did not want to work in corrections $(n=146,30 \%)$ and a few respondents said that they did not think working in corrections was important $(n=3$, $1 \%)$. Music therapists who reported working in corrections $(n=52)$ most often said they did so because it offered an opportunity to improve the lives of clients $(n=37$, $73 \%)$ or because it offered professional challenges $(n=34,67 \%)$. Some music therapists reported that they were at the beginning of their careers as music therapists and needed the experience $(n=10,20 \%)$ or that their work in corrections was an expression of their religious beliefs $(n=4,8 \%)$. About one third of respondents $(n=17$, $33 \%)$ mentioned other reasons for working in corrections. Responses from those participants who wrote in reasons for working in corrections are summarized in Table 2. The researchers grouped the "other" responses participants wrote into the following three categories: desire to work with the population, circumstantial reasons, and preferable to working with an alternative population.

When asked about political affiliation, $17 \%(n=9)$ of participants who reported working in corrections did not select a response. Of those who did select their political affiliation ( $n=43)$, the majority $(72 \%, n=31)$ reported they were Democrats, whereas $21 \%(n=9)$ were Independent and $7 \%(n=3)$ were Republicans. Most of the participants $(n=50)$ who responded to the question "To what degree do you believe your religious and/or spiritual views influence your decision to work in correctional/ forensic settings?" selected "not at all" $(52 \%, n=26)$, whereas fewer participants reported that their religious and/or spiritual views somewhat $(26 \%, n=13)$ or greatly $(22 \%, n=11)$ influenced their decision to work in corrections. Two participants who reported working in corrections did not respond to the question about religious and/ or spiritual views.

\section{Research Question 3: Practice}

The music therapists who reported which goals they addressed when working in correctional settings $(n=51)$ most frequently selected emotional awareness $(n=48$, $94 \%)$, impulse control ( $n=46,90 \%)$, self-awareness $(n=44,86 \%)$, and relaxation 
Table 2

Other Reasons for Working in Corrections

\begin{tabular}{|c|}
\hline Other reasons for wanting to work in corrections: Representative quotes \\
\hline Desire to work with the population $(n=5)$ \\
\hline $\begin{array}{l}\text { "I believe that reintegration into society presents challenges in our culture, and often these patients } \\
\text { and/or inmates are not given the tools and support that is needed to be successful. I feel that I can } \\
\text { help make a difference within the practice of music therapy." }\end{array}$ \\
\hline “Observable/Measurable growth. Charismatic population." \\
\hline “I have a passion for helping underprivileged women." \\
\hline $\begin{array}{l}\text { "I genuinely like adolescents, and I think that for urban youth, one person (or one group of people) can } \\
\text { make a difference." }\end{array}$ \\
\hline "It offers the opportunity to begin a catalyst for change for our communities." \\
\hline Circumstantial reasons $(n=6)$ \\
\hline “The opportunity came up and I took it-it's just 2 hours a week." \\
\hline "Wanted to enter federal employment." \\
\hline "Initially wanted a job in psych and prison was the only opening." \\
\hline $\begin{array}{l}\text { "I started to look for a job in a mental health program. The place I got an offer was in a correctional set- } \\
\text { ting." }\end{array}$ \\
\hline "Was transferred to Forensics division due to downsizing of hospital." \\
\hline “Company I worked for got a contract." \\
\hline Preferable to working with an alternative population $(n=2)$ \\
\hline “Needed a change from Cancer Care and Hospice." \\
\hline
\end{tabular}

and coping skills ( $n=84 \%$ ). The most frequently reported musical experiences used to address goals in correctional settings were listening to music $(n=49,96 \%)$, discussion ( $n=48,94 \%)$, playing instruments $(n=47,92 \%)$, and improvisation $(n=44$, $86 \%$ ). When asked about treatment models, music therapists working in corrections most often reported using the behavioral approach $(n=30,59 \%)$ or clinical improvisation $(n=28,55 \%)$.

\section{Research Question 4: Protocols}

\section{Facilities, Clients, and Security}

About half of participants $(n=26,50 \%)$ worked in state prisons, whereas fewer participants worked in juvenile detention centers $(n=14,27 \%)$, jails $(n=6,12 \%)$, reentry programs $(n=5,10 \%)$, transition centers $(n=3,6 \%)$, or federal prisons $(n=2$, $4 \%)$. All participants $(n=51,100 \%)$ reported that they worked with people who had substance use disorders, and more participants reported working with male inmates ( $n$ $=50,98 \%)$ than female inmates $(n=25,49 \%)$. Most respondents reported that they worked with convicted sex offenders $(n=45,87 \%)$ and more than half worked with veterans $(n=29,57 \%)$.

Almost half $(n=22,43 \%)$ of participants reported that music therapy had only existed at their facility for 1 to 5 years, although there was also a sizeable number of music therapists who reported music therapy had existed at their facility for 16 or more years $(n=14,28 \%)$. Music therapists who worked in correctional settings most frequently reported that they were always accompanied by security/staff members ( $n$ $=25,48 \%)$ or that there was a security person nearby $(n=18,35 \%)$. Of the participants who said that security personnel were not in the room or nearby, some had a beeper or phone where they could call for help if needed ( $n=15,29 \%)$, whereas oth- 
ers said their sessions were monitored electronically ( $n=8,15 \%)$. Only $8 \%(n=4)$ of participants who worked in corrections reported that they were alone in the room with inmates with no security or monitoring.

\section{Referrals and Termination of Treatment}

Social workers frequently referred individuals in correctional facilities for music therapy services $(n=29,59 \%)$ and terminated music therapy services $(n=10,39 \%)$. In many instances, a referral from a staff member was not necessary; instead, inmates could self-refer ( $n=24,49 \%)$, everyone received music therapy services $(n=22$, $45 \%)$, or the music therapist referred individuals for music therapy services $(n=21$, $43 \%)$. Less frequently, referrals came from physicians $(n=15,31 \%)$, correctional officers ( $n=9,18 \%)$, re-entry coordinators $(n=7,14 \%)$, and chaplains $(n=5,10 \%)$. Similarly, physicians $(n=6,23 \%)$, re-entry coordinators $(n=7,27 \%)$, and correctional officers $(n=5,19 \%)$ terminated sessions less often than social workers.

\section{Session Type, Group Size, and Frequency and Length of Sessions}

Most music therapists provided services in corrections both in groups and with individuals $(n=35,67 \%)$, and groups tended to have $6-10$ people $(n=30,58 \%)$ or $11-15$ people $(n=10,19 \%)$. Music therapists who provided one-on-one sessions tended to see their individual clients once $(n=22,54 \%)$ or twice $(n=12,30 \%)$ a week and sessions tended to be 30 minutes $(n=12,31 \%)$ or 45 minutes $(n=16,41 \%)$ in length. The number of individuals served per week varied greatly, but tended to be between 21 and $40(n=22,46 \%)$ or between 41 and $50(n=16,33 \%)$.

\section{Discussion}

The purpose of this study was to identify the philosophies, practices, and protocols of music therapists working in United States prisons. We used Codding's (2002) study as a point of comparison over the last 20 years and to establish a more thorough picture of today's music therapist in the United States prison setting. Finally, we used this data to present a platform for identifying areas of future research and implications for music therapy in this limited, yet growing, area of practice.

\section{Research Question 1: Demographic Variables}

\section{Gender}

The results of this survey revealed differences in demographics for music therapists who worked in prisons. Among music therapists who reported they did not work in prisons, females outnumbered males by almost 8 to 1 , whereas female music therapists working in prisons only outnumbered male music therapists working in prisons by about 3 to 1 . The demographic variables of music therapists who reported they did not work in prisons reflect the gender ( $88 \%$ female) and ethnicity ( $87 \%$ white) of music therapists who responded to AMTA's 2018 workforce analysis (American Music Therapy Association, 2018). The gender distribution of music therapists in the present study who reported working in corrections was also similar to that reported in Codding's 2002 study. These similarities suggest that little has changed in the demographic composition of both music therapists working in corrections and in the field of music therapy as a whole.

The overrepresentation of male music therapists employed in the correctional setting could be related to the overall demographics of correctional employees. State, federal, and private prisons tend to employ more male than female security staff (Burdkhard, 2017) and working in a setting where there are few other women (as employees and as inmates; Carson, 2020; Federal Bureau of Prisons, 2021) could be intimidating for some female music therapists. 
Current survey results suggest that many music therapists would be open to working in the correctional settings if job opportunities existed. This may also be a cue to academic programs who may not (or may be unable to) include corrections as part of their experiential learning component. For music therapists in training, the opportunity to see the value of music therapy services within this population, and to experience the potential demystification of the prison population, may allow students to see how they fit in working in this setting.

\section{Political Affiliation}

Questions about political affiliation and religious beliefs are apropos to research on workers in correctional facilities, since previous researchers have found that both factors may influence attitudes about criminal punishment (Thomson \& Froese, 2017). In the present study, there were more music therapists working in corrections who identified as Democrats and the majority of respondents indicated that their religious beliefs or spiritual views did not influence their decision to work in corrections at all. It was important to know the spiritual and/or religious views of participants where this influenced their decision to work in corrections. Awareness of these religious motivations for providing evidence-based practice in this setting is important in an effort to maintain professional boundaries and to objectively deliver music therapy services.

It is interesting that very few music therapists worked in local jails, even though there are roughly 3,000 local jails in the U.S. that outnumber other types of correctional facilities (Sawyer \& Wagner, 2020). Approximately half of participants in the present study worked in state prisons and a quarter worked in juvenile detention centers. It is possible that the purpose and function of jails or lack of resources at the local level make it more challenging for jails to employ music therapists.

\section{Research Question 3: Practice}

\section{Goals}

In setting goals, most music therapists focused on issues presented by people with substance abuse disorders, sex offenders, or individuals who have served in the military. These areas align with existing literature examining the needs of those incarcerated (Carson, 2020; Fazel et al., 2017). In the present study, music therapists reported most frequently addressing the goals of emotional awareness, impulse control, self-awareness, or relaxation and coping skills when working in correctional settings.

The behavioral approach and clinical improvisation techniques remain consistent as the most commonly used intervention practice among music therapists as evidenced by the results of the current study, and in comparison to Codding's (2002) study nearly two decades ago. This could suggest that music therapy training programs that emphasize these approaches may be especially equipped to offer practica for their students with this population, and currently practicing clinicians who have experience in this approach to treatment may be particularly equipped to offer services. It may also be the case that these approaches are continually used as they are effective in treating common diagnoses of prisoners.

Music therapists also reported that they primarily deliver music therapy services in a group format. These results are valuable to know in that the existing research and the current practice seem to reinforce the efficacy of the treatment goals as evidenced by their repeated use and successful outcomes. It would be interesting to further examine why these trends occur and for what reason. For example, is the group format conducive for helping to prepare for service provision for music therapists? Additionally, these goals are similar to those of other populations with whom music therapists often work and in contexts such as behavioral health centers. While each population has inherent differences, understanding the similarities can be valuable as they allow clinicians to offer treatment to a variety of populations and settings or to enhance the services they already offer. 


\section{Research Question 4: Protocols}

Several practices and protocols reported by music therapists in the present study (all of whom work in the U.S.) were different from those reported by Gold et al. (2014), whose research took place at a correctional facility in Norway. Respondents to our survey reported that they often used music listening, discussion, instrument play, or improvisation; this is in contrast to the most frequently used interventions reported by Gold et al. (2014), which were structured music interplay, singing, and verbal reflection. The music therapist in the study by Gold et al. (2014) reported a greater frequency of sessions (2-3/week, compared to 1-2/week in our study), and smaller groups (1-6 people, compared to 6-10 people in our study). It is unclear the degree to which these differences are due to the uniqueness of the correctional system in the U.S. (which incarcerates more people per capita than any other country). It could be speculated that these differences exist due to varying cultural perceptions of what it means to be incarcerated. It could also be reflective of the fact that the U.S. is singularly phenomenal in its treatment and judgement of criminals and these differences are further highlighted when compared to countries around the world. The centuries old U.S. treatment models of imprisonment that largely emphasize a punitive treatment model make implementing creative rehabilitative services such as music therapy challenging, but not impossible. It must be mentioned, however, that there are correctional facilities, organizations, and members of correctional leadership who value, promote, and support the use of music therapy in the rehabilitation of its inmates.

\section{Implications for Clinical Practice}

Since results indicate that several new music therapy programs have been established in correctional facilities in the U.S. in recent years, the information about music therapy practices in corrections presented in this study may be helpful for music therapists who are seeking to establish new programs in correctional facilities. Music therapists establishing new programs in correctional facilities in the U.S. should form strong working relationships with the social workers at their facilities, since participants in the present study reported that social workers were most likely to refer clients for music therapy services. The prevalence of group work for music therapists working in corrections indicates that music therapists hoping to work in corrections should have ample training and experience understanding and managing group dynamics.

Although we examined the philosophies of music therapists working within the U.S. correctional system, we recommend that music therapists better understand the philosophies of those who make broader policy and administrative decisions related to the criminal justice system in their country. Understanding how the U.S. criminal justice system works (and when, why, and for whom it does not work) can better help music therapists understand and connect with the incarcerated clients they serve and their family members. Furthermore, understanding the broader context of incarcerated clients' experiences can help music therapists advocate for the rights of their clients at the individual, institutional, and policy levels, no matter where they live. Given the inherent power dynamics that exist within the relationship between music therapists and their clients who are incarcerated, music therapists working in or preparing to work in corrections should carefully consider how aspects of their identity and political/religious beliefs might influence their clinical practice, and take steps to improve their self-awareness, knowledge, and skills in order to provide culturally competent care.

\section{Limitations}

Several limitations may have influenced the outcomes of this study. Due to the small sample size, it is difficult to determine the true response rate among music therapists who work in corrections. The overall response rate (9\%) includes music therapists who did not work in corrections. It is possible that some music therapists who did not work in corrections chose not to participate because they read the study title and thought 
they were ineligible to participate. The wording of certain questions in the survey may have caused confusion for the participants and minimized the number of people appropriate for taking the survey. For example, one question asked participants, "Do you practice music therapy in a prison?" Those who answered "no" were thanked for their participation and the survey ended. This question would have excluded respondents working with individuals who are under correctional supervision, but residing outside of a prison setting. It is not clear to what degree the information collected reflects practice of all music therapists in the U.S. working in correctional facilities. Because of these limitations, music therapists working in other countries should take caution when generalizing results from this study to their practice.

Additionally, asking respondents to make generalizations about their previous work can dilute the accuracy of their reporting. What music therapists are actually doing in their daily routine may be different than the perceptions of what is actually happening. In this survey, for example, participants were asked to identify how many people were in their sessions and how long their sessions lasted; it is not clear how accurate their estimates are.

\section{Future Research}

Existing literature supports the efficacy of music therapy in this setting and information regarding the music therapists who work within these settings is lacking. Knowing more about the relatively small workforce of therapists working in prisons is valuable so that the future of music therapy in prisons has a pathway for growth, success, and evidence-based outcomes. As a result of the information gathered from this survey, a plethora of future areas of research presents itself. It would be of benefit to know how demographics, religion, race, and gender impact each of these elements, how those experiencing incarceration respond to services, and in what ways these dynamics impact the daily functioning of a music therapist in this setting. Exploring the benefits to those post-incarceration and transitioning into civilian life could offer meaningful insight. For example: halfway houses, transition programs, or substance abuse/behavioral health settings might offer meaningful comparisons that can be helpful in further understanding the correctional setting. Conducting research in a prison is difficult at best and if conducting research in a parallel, civilian-like setting can offer insight that can be transferred to the incarcerated population, that would be valuable. Hopefully, this study initiates many more research studies in the future.

\section{About the Authors}

Lorna E. Segall, Assistant Professor of Music Therapy, directs the music therapy program at the University of Louisville. Her areas of research interest include music therapy in corrections, intergenerational programming, and the aging population.

Olivia Swedberg Yinger, Associate Professor of Music Therapy, directs the music therapy program at the University of Kentucky. She serves as Associate Editor for the Journal of Music Therapy. Her research interests include trauma-informed music therapy and pediatric procedural support.

\section{References}

Al-Rouson, T., Rubenstein, L., Sieleni, B., Deol, H., \& Wallace, R. B. (2017). Inside the nation's largest mental health institution: A prevalence study in a state prison system. BMC Public Health, 17(1), 342. https://doi.org/10.1186/s12889-017-4257-0

Allard, T. J., Wortley, R. K., \& Stewart, A. L. (2003). Role conflict in community corrections. Psychology, Crime \& Law, 9(3), 279-289. https://doi.org/10.1080/1068316031000093414

American Music Therapy Association. (2006). Music therapy for persons in correctional and forensic settings. https://www.musictherapy.org/assets/1/7/MT_Forensics_2006.pdf 
American Music Therapy Association. (2018). 2018 AMTA member survey and workforce analysis: A descriptive, statistical profile of the 2018 AMTA membership and the music therapy community. American Music Therapy Association.

Beall, S. K. (2018). Lock her up!: How women have become the fastest growing population in the carceral state. Berkeley Journal of Criminal Law, (23)2. https://doi.org/10.15779/ Z38X34MS09

Burkhardt, B. C. (2017). Who is in private prisons? Demographic profiles of prisoners and workers in American private prisons. International Journal of Law, Crime and Justice, 51, 24-33. https://doi.org/10.1016/j.ijlcj.2017.04.004

Carson, E. A. (2020, October 22). Prisoners in 2019 (NCJ 255115). U.S. Department of Justice, Office of Justice Programs, Bureau of Justice Statistics. https://www.bjs.gov/ index.cfm?ty $=$ pbdetail\&iid $=7106$

Carson, E. A., \& Sabol, W. J. (2016, May 19). Aging of the state prison population, 1993-2013 (NCJ 248766). U.S. Department of Justice, Office of Justice Programs, Bureau of Justice Statistics. https://www.bjs.gov/index.cfm?ty $=$ pbdetail\&iid $=5602$

Chen, X., Hannibal, N., \& Gold, C. (2015). Randomized trial of group music therapy with Chinese prisoners: Impact on anxiety, depression, and self-esteem. International Journal of Offender Therapy and Comparative Criminology, 60(9), 1064-1081. https://doi.org/10.1177/ $0306624 X 15572795$

Chen, X., Leith, H., Aarø, L. E., Manger, T., \& Gold, C. (2016). Music therapy for improving mental health problems of offenders in correctional settings: Systematic review and metaanalysis. Journal of Experimental Criminology, 12(2), 209-228. https://doi.org/10.1007/ s11292-015-9250-y

Codding, P. A. (2002). A comprehensive survey of music therapists practicing in correctional psychiatry: Demographic conditions of employment, service provision, assessment, therapeutic objectives, and related values of the therapist. Music Therapy Perspectives, 20(2), 56-68. https://doi.org/10.1093/mtp/20.2.56

Cohen, J. M. (1987). Music therapy with the overcontrolled offender: Theory and practice. The Arts in Psychotherapy, 14(3), 215-221. https://doi.org/10.1016/0197-4556(87)90004-9

Daveson, B. A., \& Edwards, J. (2001). A descriptive study exploring the role of music therapy in prisons. The Arts in Psychotherapy, 28(2), 137-141. https://doi.org/10.1016/ S0197-4556(00)00089-7

Denney, A. S. (2017). Prison chaplains: Inmate/correctional officer role perceptions and conflict management in modern corrections. Corrections, 2(3), 189-210. https://doi.ort/ 10.1080/23774657.2017.1291315

Dolan, M. (2020). The first step act's misstep: Why the first step act violates prisoners' rights to equal protection. American University Law Review Forum, 69(6), 233-265.

http://www.aulawreview.org/the-first-step-acts-misstep-why-the-first-step-act-violatesprisoners-rights-to-equal-protection/

Evans, T. D., \& Adams, M. (2003). Salvation or damnation?: Religion and correctional ideology. American Journal of Criminal Justice, 28, 15-35. https://doi.org/10.1007/ BF02885750

Fazel, S., Yoon, I. A., \& Hayes, A. J. (2017). Substance use disorders in prisoners: An updated systematic review and meta-regression analysis in recently incarcerated men and women. Addiction, 112(10), 1725-1279. https://doi.org/10.1111/add.13877

Federal Bureau of Prisons. (2021). Inmate gender. Federal Bureau of Prisons: United States Government. https://www.bop.gov/about/statistics/statistics_inmate_gender.jsp

Field, A. (2013). Discovering statistics using IBM SPSS statistics (4th ed.). Sage Publications.

Formerly Incarcerated Reenter Society Transformed Safely Transitioning Every Person Act of 2018, Pub. L. No. 115-391, 132 Stat. 5194 (2018). https://www.congress.gov/115/plaws/ publ391/PLAW-115publ391.pdf 
Gold, C., Assmus, J., Hjørnevik, K., Qvale, L. G., Brown, F. K., Hansen, A. L., Waage, L., \& Stige, B. (2014). Music therapy for prisoners: Pilot randomised controlled trial and implications for evaluating psychosocial interventions. International Journal of Offender Therapy and Comparative Criminology, 58(12), 1520-1539.https://doi.org/10.1177/ $0306624 X 13498693$

Gold, C., Due, F. B., Thieu, E. K., Hjørnevik, K., Tuastad, L., \& Assmus, J. (2020). Long-term effects of short-term music therapy for prison inmates: Six-year follow-up of a randomized controlled trial. International Journal of Offender Therapy and Comparative Criminology. Advance online publication. https://doi.org/10.1177/0306624X20909216

Hakvoort, L., Bogaerts, S., Thaut, M. H., \& Spreen, M. (2015). Influence of music therapy on coping skills and anger management in forensic psychiatric patients: An exploratory study. International Journal of Offender Therapy and Comparative Criminology, 59(8), 810-836. https://doi.org/10.1177/0306624X13516787

Hjørnevik, K., \& Waage, L. (2019). The prison as a therapeutic music scene: Exploring musical identities in music therapy and everyday life in a prison setting. Punishment \& Society, 21(4), 454-472. https://doi.org/10.1177/1462474518794187

Jacobsen, J., Heard, C., \& Fair, H. (2017). Prison: Evidence of its use and over-use from around the world. Institute for Criminal Policy Research. https://www.prisonstudies.org/sites/ default/files/resources/downloads/global_imprisonment_web2c.pdf

Maahs, J., \& Pratt, T. (2001). Uncovering the predictors of correctional officers' attitudes and behaviors: A meta-analysis. Corrections Management Quarterly, 5(2), 13-19.

Nolan, P. (1983). Insight therapy: Guided imagery and music in a forensic psychiatric setting. Music Therapy, 3(1), 43-51. https://doi.org/10.1093/mt/3.1.43

O'Grady, L., Rolvsjord, R., \& McFerran, K. (2015). Women performing music in prison: An exploration of the resources that come into play. Nordic Journal of Music Therapy, 24(2), 123-147. https://doi.org/10.1080/08098131.2013.877518

Odell-Miller, H., Bloska, J., Browning, C., \& Hannibal, N. (2019). Process and experience of change in the self-perception of women prisoners attending music therapy: The qualitative results of a mixed-methods exploratory study. Approaches: An Interdisciplinary Journal of Music Therapy. Advance online publication. http://approaches.gr/wp-content/uploads/ 2019/08/Approaches_FirstView_a20190805-odell-miller.pdf

Porter, N. D. (2020). Top trends in criminal justice reform. The Sentencing Project. https://www.sentencingproject.org/publications/top-trends-in-state-criminal-justicereform-2019/

Sawyer, W., \& Wagner, P. (2020, March 24). Mass incarceration: The whole pie. Prison Policy Initiative. https://www.prisonpolicy.org/reports/pie2020.html

Segall, L. E. (2016). The effect of a music therapy intervention on inmate levels of executive function and perceived stress: Teaching for transfer [Doctoral dissertation, Florida State University]. DigiNole: FSU's Digital Repository. https://fsu.digital.flvc.org/islandora/object/ fsu\%3A366121

Silverman, M. J., \& Furman, A. G. (2014). Employment and membership trends in the American Music Therapy Association, 1998-2009. Music Therapy Perspectives, 32, 99-108. https://doi.org/10.1093/mtp/miu009

Sims, B. (2003). The impact of causal attribution on correctional ideology: A national study. Criminal Justice Review, 28(1), 1-25. https://doi.org/10.1177/073401680302800102

Sundt, J. L., \& Cullen, F. T. (2002). The correctional ideology of prison chaplains: A national survey. Journal of Criminal Justice, 30(5), 369-385. https://doi.org/10.1016/ S0047-2352(02)00152-6

Thielo, A. J., Cullen, F. T., Cohen, D. M., \& Chouhy, C. (2015). Rehabilitation in a red state: Public support for correctional reform in Texas. Criminology \& Public Policy, 15(1), 137-170. https://doi.org/10.1111/1745-9133.12182 
Tuastad, L. \& O'Grady, L. (2013). Music therapy inside and outside prison-A freedom practice? Nordic Journal of Music Therapy, 22(3), 210-232.https://doi.org/10.1080/ 08098131.2012.752760

Tuastad, L., \& Stige, B. (2015). The revenge of Me and THE BAND'its: A narrative inquiry of identity constructions in a rock band of ex-inmates. Nordic Journal of Music Therapy, 24(3), 252-275. https://doi.org/10.1080/08098131.2014.967713

Thaut, M. H. (1989). The influence of music therapy interventions on self-rated changes in relaxation, affect, and thought in psychiatric prisoner-patients. Journal of Music Therapy, 26(3), 155-166. https://doi.org/10.1093/jmt/26.3.155

Thomson, R. A., \& Froese, P. (2017). God versus party: Competing effects on attitudes concerning criminal punishment, national security, and military service. Journal for the Scientific Study of Religion, 55(4), 839-858. https://doi.org/10.1111/jssr.12293

van de Wall, W. (1923). Music in correctional institutions. J. B. Lyon Company.

Vasquez, M. J. T. (2007). Cultural differences and the therapeutic alliance: An evidence-based analysis. American Psychologist, 62(8), 878-885. https://doi.org/10.1037/ 0003-066X.62.8.878 